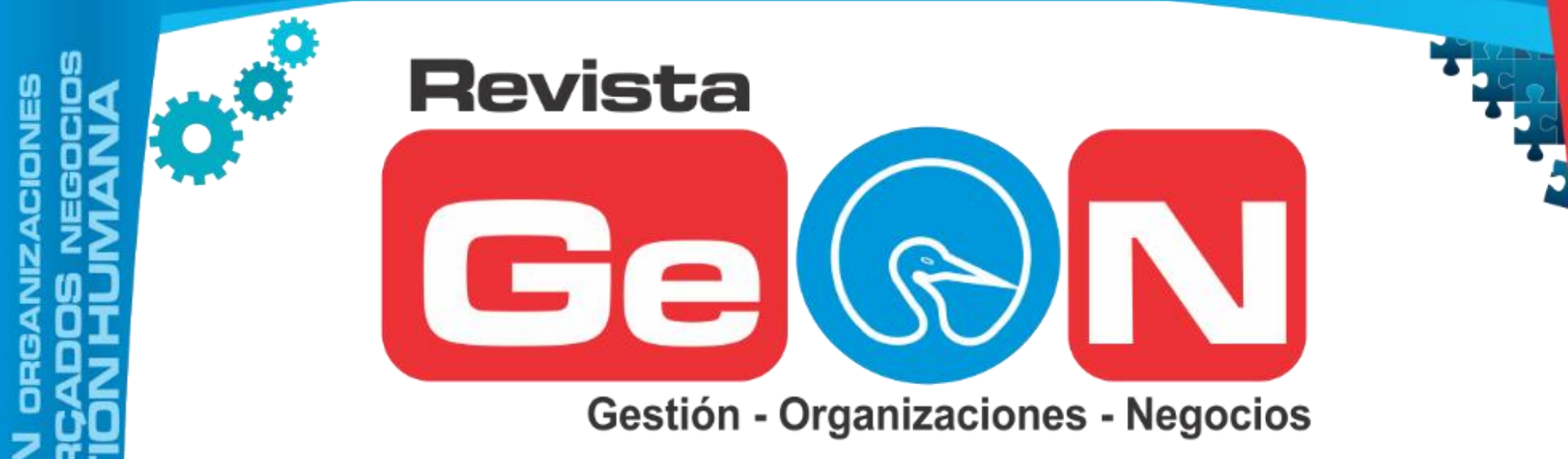

2 it ISEN EC4E - 3910 Volumén E No 1 Enero a Junio E015

Revista Electrónica de la Facultad de Ciencias Económicas de la Universidad de los Llanos

는 Revista Electronica de la FaculaVICENCIO- COLOMBIA

$4 \frac{4}{2}$

04

72

21

- 2

단

(i)

ii III

(ㄴ)

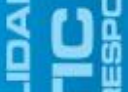

IiI
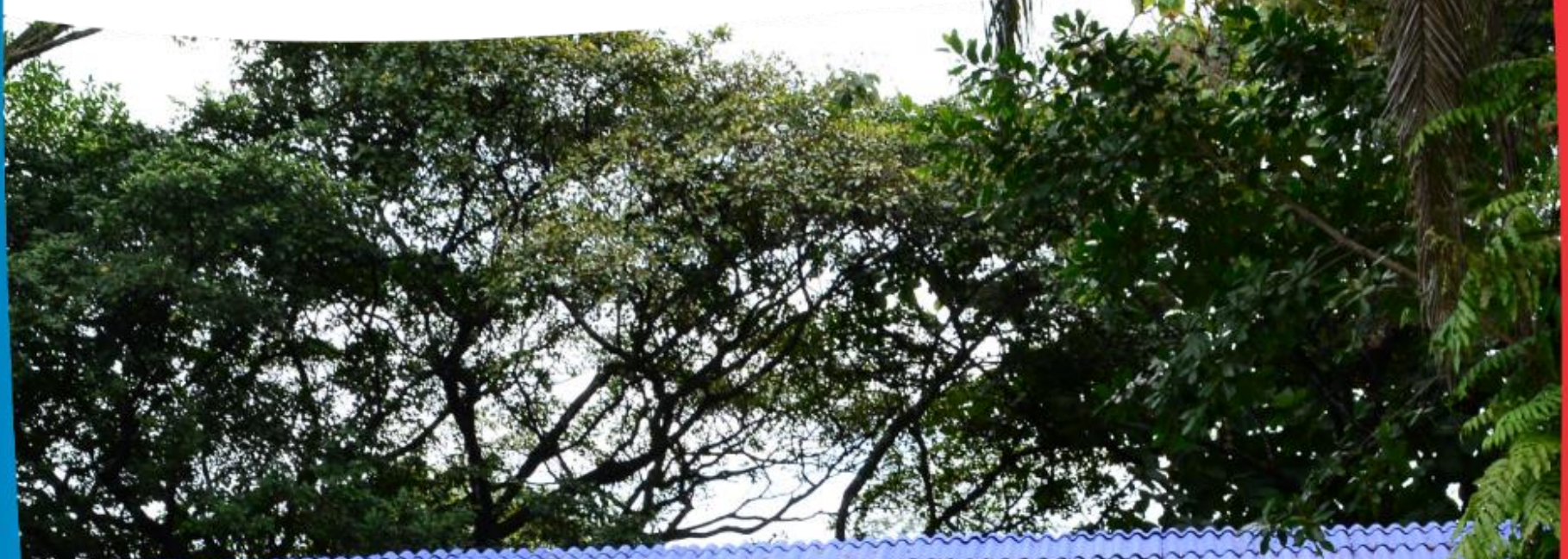

III) in 0

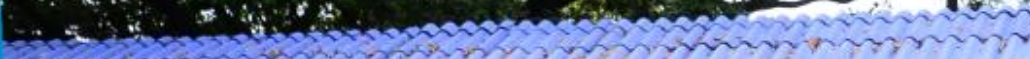

0

iii

î. II

40

82

T

80

更 II

3

,

2

20

QN

42

04

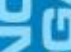

5 iा

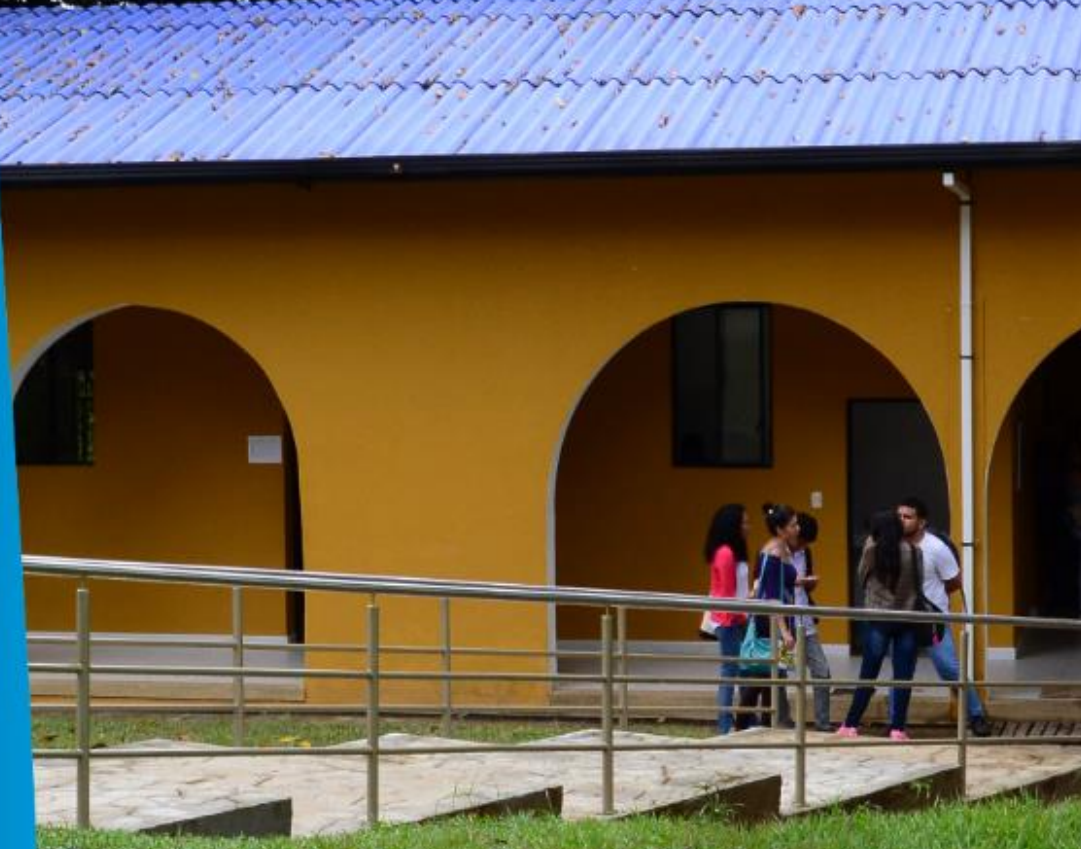

-

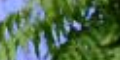




\section{Rediseño administrativo y organizacional para la Comercializadora $4 \times 4$ en la ciudad de Villavicencio - por Yessica Alejandra Benitez Barrera.}

Yessica Alejandra Benitez Barrera

Administradora de Empresas

yeabeba2305@hotmail.com

@yessicabenitez7

\section{Resumen}

Este artículo presenta los resultados obtenidos en el desarrollo de la investigación realizada a la Comercializadora $4 \times 4$, microempresa de la ciudad de Villavicencio. $\mathrm{Su}$ objetivo fue rediseñar la estructura administrativa y organizacional de la comercializadora $4 \times 4$ de modo que se fortalezcan los procesos y se direccione la empresa hacia la ampliación del mercado actual.

Tanto la propuesta como el desarrollo de la investigación se enmarcaron dentro de teorías administrativas que direccionaron la elaboración de un diagnóstico completo de la organización, la formulación de una plataforma estratégica y la propuesta de rediseño organizacional orientada hacia los procesos, los cuales se caracterizaron por la recopilación de información, actualización y mecanismos de mejora. El desarrollo de la investigación involucró la participación del personal de la Comercializadora $4 \mathrm{X} 4$ y la asesoría de especialistas de la Universidad de Los Llanos.

\section{Palabras Clave}

Calidad, procesos, organización, administración, implementación, estrategia, innovación, tecnología.
Abstract
This paper presents the results found in the development of the research made to "Comercializadora 4x4", microenterprise from Villavicencio Meta. Its objective was redesign the administrative and organizational structure of the enterprise such that the processes be

strengthened and the company be routed to the broadening of its market.

As well proposal as development of the research were performed within administrative theories wich routed the elaboration of a complete diagnostic from the company, the formulation strategic platform and the proposal of a organizational redesign directed to the processes. Investigation involved the participation from enterprise staff and consulting from specialist of the Llanos University.

\section{Keywords}

Management; strategy; quality; technology; innovation; administration; processes; implementation.

\section{Introducción}

El mercado ante el cual se enfrenta en la actualidad toda organización es mucho más competitivo que el de hace unas décadas, la empresa que quiera mantenerse vigente en estos tiempos debe presentar un sistema flexible $\mathrm{y}$ abierto al cambio, y debe mantenerse en constante innovación para que los servicios y productos sobresalgan ante los demás.

La globalización se ha encargado de poner al alcance de todas las organizaciones, sean estas grandes, medianas o pequeñas, los mismos recursos competitivos, lo que las confronta a mayor competencia y cambios del mercado, contexto en el que una empresa que no innove y sobresalga no sobrevivirá.

Una empresa no puede aspirar al liderazgo del mercado a menos que haya edificado recursos organizativos y humanos para hacerse competitiva y sus ejecutivos sean lo suficientemente visionarios y flexibles para revisar constantemente sus objetivos y actualizarlos. Estos principios se hacen más pertinentes a medida que la organización desee 
alcanzar crecimiento y posicionamiento en un nicho de mercado determinado.

Por esta se razón, se pretende hacer un diagnóstico al estilo de planeación, dirección, organización y control de la Comercializadora $4 \times 4$ y de acuerdo a este, plantear el modelo organizacional y administrativo idóneo que la lleven al planteamiento de objetivos y enfoquen su crecimiento en el mercado en el que se desenvuelven.

\section{Desarrollo}

Se realizaron 4 procesos de recolección de información para la construcción de la matriz DOFA. Estos procesos previamente planeados en la metodología de la investigación fueron la base para contextualizar el entorno organizacional y situación actual de las diferentes áreas de trabajo de la Comercializadora $4 \times 4$.

En la visita se analizó cualitativamente las características visibles de la organización y se indagó sobre los inicios de esta. En la reunión con el personal se abrió un espacio de opinión acerca de las condiciones actuales de cada área de la empresa mediante una entrevista semiestructurada enfocada al conocimiento del modelo administrativo actual y los procesos llevados a cabo dentro de la organización.

Posteriormente se realizó la construcción de las matrices PCI y POAM junto con la gerencia y el personal de ventas, quienes fueron los encargados de asignar una calificación a cada factor de estas y trabajaron en conjunto con la investigadora en el análisis de estas variables.

Una vez se identificaron los factores $\mathrm{y}$ variables de mayor impacto en la organización, se procedió a realizar un análisis de vulnerabilidad y competitividad de la empresa a través de las cinco fuerzas de PORTER que permitieron estar al tanto del impacto de la empresa en su entorno y mercado actual.
Finalizando los anteriores procesos se realizó una matriz DOFA que recopila los datos de más relevancia en todos los análisis y dio paso a la creación de estrategias para el mejoramiento y aprovechamiento de todos los factores inmersos en la organización.

\section{Visita a la empresa}

De esta manera se encontraron 5 factores claves del éxito:

1. Diseño organizacional. Es Prioritario que la empresa fije canales de autoridad, responsabilidad y control a cada uno de sus procesos, para que de esta manera todo esfuerzo o labor sea encaminada a la consecución de los objetivos organizacionales.

2. Personal comprometido. El talento humano debe estar comprometido en el cumplimiento de sus labores, en la satisfacción del cliente, en la innovación y para que esto sea posible la organización debe abrir canales de comunicación que le permitan a este trabajar de manera propositiva. Adicional se debe crear un programa de incentivos y capacitaciones permanentes encaminadas a la mejora continua de cada uno de los procesos. Un ejemplo de cómo se compromete el personal.

3. Implementación y uso continúo de TIC'S. $\mathrm{Si}$ la empresa quiere alcanzar altos niveles de efectividad en todas sus áreas y no incurrir en gastos permanentes de personal adicional, debe hacer uso continuo de la tecnología en todas sus operaciones.

4. Innovación constante. Al tener un alto número de competidores y productos sustitutos se hace necesario generar valor agregado que diferencie a la organización de otras empresas en el mercado.

5. Investigación en mercadotecnia. A pesar de que la organización cuenta actualmente con un personal altamente efectivo en el área de ventas, no se hace uso completo de todos los elementos de investigación en el área de mercados y por tanto se está dejando pasar la 
oportunidad de ampliar el mercado para sus diferentes productos.

Posteriormente se construyó la plataforma estratégica que según lo expuesto por Mejía (2006),

[...] constituye la carta de navegación, el derrotero por donde se desplazara la empresa, esa carta de navegación debe construirse de manera democrática y participativa, y siempre debe considerarse la necesidad de disponer de ella, además del acceso para todos, debe editarse y reproducirse, elaborar un plan de educación y capacitación, no debe haber un solo actor que no conozca la cara fundamental de la empresa ( $\mathrm{p}$. 40).

También se definieron la misión, visión, objetivos organizacionales y valores o principios organizacionales, a saber:

Misión. Somos una empresa comercializadora de productos alimenticios de calidad para la satisfacción de nuestros clientes, con procesos en mejora continua que sean de agrado a nuestros consumidores, de eficiente gestión, competitiva, con alianzas estratégicas a nivel regional, comprometida con el servicio al cliente, la formación integral de su recurso humano y tecnológico.

Visión. La Comercializadora $4 \times 4$ será al 2020 una empresa posicionada y reconocida en todo el departamento, brindando al cliente productos y servicios de excelente calidad, inspirados en un modelo innovador.

Objetivos organizacionales. Estos serán los resultados específicos y mesurables de lo que la empresa desea alcanzar:

1. Impulsar la excelencia, productividad y competitividad de la organización mediante una innovación continua en los procesos internos y externos.

2. Promocionar el nombre de la empresa generando identificación, visibilidad social y económica en cada uno de sus productos.

3. Promover la investigación y el desarrollo tecnológico en sus diferentes procesos a través del análisis, la evaluación y la difusión de las mejores prácticas e innovaciones actuales.
4. Aumentar la cobertura de los diferentes nichos de mercado para sus productos dentro de la región.

5. Generar valor agregado a sus productos con gran número de sustitutos en el mercado mediante la investigación y la tecnología.

En cuanto a los principios corporativos, la Comercializadora $4 \times 4$ los define como la base de cada acción llevada a cabo en la empresa, a saber, efectividad, calidad, compromiso, eficiencia, responsabilidad

En cuanto a sus valores organizacionales, éstos deben ser conocidos y aplicados por toda la organización: honestidad, verdad, responsabilidad, solidaridad, respeto, compromiso.

\section{Identificación de procesos}

Dentro de la organización se identificaron tres procesos básicos de los cuales se desprenden todas las labores de la organización: transformación, administración, mercadeo.

Con base en los hallazgos procedentes de la investigación a la Comercializadora $4 \times 4$, se determinó que la empresa necesita definir un modelo estructural y administrativo que la haga más ágil, competitiva y presente un sistema de toma de decisiones descentralizado que abarque todas las áreas de la organización.

Se hace necesario que la organización optimice las operaciones que se llevan cabo dentro de ella y asigne cadenas de responsabilidad, autoridad y valor en todos sus niveles; observación que se deriva de la falta comunicación y participación del personal en la toma de decisiones.

Se buscó un modelo organizacional que dirija el talento humano hacia la consecución de los objetivos generales, que genere valor en cada área de trabajo, que vaya en función de la satisfacción del cliente y lo que este requiere, que posea un sistema de comunicación interna eficiente y que implique a todo el personal que compone la organización hacia la visión de esta.

Por esta razón se decidió plantear un modelo organizacional por procesos, con la que, según 
Muro (2014) "[...] organice la empresa alrededor de las actividades que generen valor agregado al cliente, independientemente del área a la que pertenezcan y que convierta al personal en el motor de la organización" (s/p). Todo esto con el fin de invitar a la mejora e innovación continua.

\section{Administración por procesos}

$\begin{array}{cccr}\text { Para la implementación de una } & \text { de } \\ \text { administración por procesos en la }\end{array}$ Comercializadora $4 \times 4$ debe cambiarse la visión del personal sobre la empresa misma y entender que el punto de partida de un mercado satisfecho será el entendimiento de la organización como una cadena de valor completa, en la que cada área depende de todas las otras para desempeñar una buena función y que si no se consigue el resultado esperado será responsabilidad de toda la empresa.

Una manera de visualizar este aspecto será que cada persona dentro de la organización recibe algo, lleva a cabo una actividad determinada con ese algo, es decir, le aporta valor y entrega algo después de su parte del proceso. Esto se denomina cadena cliente interno - proveedor interno. De esta manera, si cada persona dentro de la empresa se orienta hacia su cliente interno, toda la empresa en conjunto se orientara hacia el cliente final (Acuña, 2012, s/p).

La atención se ha de centrar en los resultados del proceso entero $\mathrm{y}$ no en las tareas $\mathrm{o}$ actividades que cada persona debe realizar, la empresa pasara de ser un conjunto de departamentos a un grupo de talento humano que hace parte de uno o varios procesos dentro de la organización.

No obstante, el éxito de la administración por procesos está supeditado a unos requerimientos por parte del personal para su implementación, a saber: a) Orientar la empresa y todas sus funciones, hacia el cliente; b) No resistencia al cambio; c) Orientación hacia resultados en conjunto; d) Rendimiento frente a los procesos y clientes en lugar a departamentos y jefes; e) Participación, responsabilidad y apoyo como base de autoridad y control; f) Eliminación de procesos que no generen valor.

Superados estos requerimientos, se puede esperar como resultado de la implementación de la gestión por procesos, los siguientes beneficios: a) Integración y alineación de los procesos para permitir el logro de los objetivos organizacionales; b) Capacidad para centrar los esfuerzos en la eficiencia y eficacia de los procesos; c) Menores costos y tiempos de ciclos más cortos, a través del uso eficaz de los recursos; d) Medición de los procesos en relación con el valor añadido percibido por el cliente; e) Establecimiento de responsables para cada proceso desarrollado; f) Cambio de mentalidad de "qué se hace" a "para quién se hace".

Acorde con los principales procesos identificados en la organización, se diseñó un diagrama por procesos para la comercializadora $4 \times 4$, en este se tiene como entrada los requerimientos del cliente, por salidas la satisfacción de este mismo.

Se tiene un grupo de procesos estratégicos cuyo fin es fortalecer los principales procesos de la organización, estos son: capacitación y motivación, generación de valor, inversión continua en investigación y tecnología y la apertura de nuevos mercados.

Adicional a estos se determinó un grupo de procesos de soporte: contabilidad y finanzas, recurso humano, mezcla de mercadotecnia, adquisición de insumos, dirección y control, mantenimiento de instalaciones y maquinas.

\section{Conclusiones}

Del estudio realizado en la Comercializadora 4X4 queda como conclusión que la empresa actualmente no refleja en ninguno de sus procesos una cadena de responsabilidad o de mando definido, no está actualizada en el ámbito 
administrativo y posee criterios de toma de decisiones totalmente centrados y autoritarios.

El personal de la organización no recibe en ninguno de sus etapas la capacitación necesaria para realizar un proceso de calidad.

La empresa se rige por la cotidianidad con el único objetivo de sobrevivir en el mercado y garantizar bienestar a sus propietarios, ya que no se tienen objetivos organizacionales y mucho menos estrategias de acción.

Desde una perspectiva de valoración profesional personal, cabe señalar que la comercializadora $4 \times 4$ es un buen ejemplo de iniciativa empresarial, que aunque con grandes falencias de gestión, denota pujanza, persistencia y gran tenacidad.

El estudio actual contribuyó, tanto en la observación, búsqueda y formulación de elementos de planeación, dirección, organización y control dentro de la empresa, con lo cual aporté criterios de mi experiencia profesional, y de igual manera motivó mi personal interés por la creación de organizaciones sostenibles y generadoras de empleo.

\section{Referencias}

Acuña, Jorge (2012). "Administración por procesos. Perspectiva tecnológica", sitio web de pnt, [en línea], disponible en: http://www.pnt.org.mx/boletin/Marzo 2012/Pdf/ Administracion_de_Procesos.pdf [accesado el día 17 de agosto de 2014].

Amaya A., Jairo (2005). "Gerencia planeación y estrategia", sitio web de books.google, [en línea], disponible en: http://books.google.com.co/books?id=8Flzg6f8d OsC\&printsec $=$ frontcover $\&$ hl $=$ es \&source $=g b s$ vpt reviews\#v=onepage $\& \mathrm{q} \& \mathrm{f}=$ false $\quad$ [accesado el día 17 de agosto de 2014].

Mejía G., Braulio (2006). Gerencia de procesos para la organización y el control interno de empresas de salud. Quinta edición. Bogotá: Eco Ediciones.

Muro, Pedro (2010). "Beneficios de la gestión por procesos. Innovación en el management desde la necesidad del cliente", sitio web de arpcalidad, [en línea], disponible en: http://www.arpcalidad.com/los-beneficios-de-lagestin-por-procesos/ [accesado el día 17 de agosto de 2014].

Vidal A., Elizabeth (2004). Diagnóstico organizacional, evaluación sistémica del desempeño empresarial en la era digital. Segunda edición. Bogotá: Eco ediciones. 